\title{
Entanglement for all quantum states
}

\author{
A. C. de la Torre* D. Goyeneche $\dagger$ and L. Leitad \\ IFIMAR - (CONICET-UNMDP) \\ Departamento de Física - Universidad Nacional de Mar del Plata \\ Funes 3350, 7600 Mar del Plata, Argentina.
}

\begin{abstract}
It is shown that a state that is factorizable in the Hilbert space corresponding to some choice of degrees of freedom, becomes entangled for a different choice of degrees of freedom. Therefore, entanglement is not a special case but is ubiquitous in quantum systems. Simple examples are calculated and a general proof is provided. The physical relevance of the change of tensor product structure is mentioned.
\end{abstract}

Keywords: entanglement, factorization, correlations, tensor product structure.

PACS: 03.65.Ca 03.65.Ud

Published in Eur. J. Phys. 31 (2010) 325-332.

\section{INTRODUCTION}

Entanglement is one of the most remarkable features of quantum mechanics. Consider two exclusive properties of a quantum system, $A_{1}$ and $A_{2}$, corresponding to two different eigenvalues of some observable (for instance, spin up and spin down) and also another unrelated pair of exclusive properties, $B_{1}$ and $B_{2}$ (for instance, located here or there). Furthermore, imagine two possible states of the system: $\psi_{1}$, corresponding to the simultaneous appearance of the properties $A_{1}$ and $B_{1}$ and the other state, $\psi_{2}$, corresponding to the appearance of the properties $A_{2}$ and $B_{2}$. The superposition, $\psi_{1}+\psi_{2}$, is an entangled state of the system. In this state, none of the properties $A_{1}, A_{2}, B_{1}, B_{2}$ are objective (in the sense that the state is not an eigenvector corresponding

\footnotetext{
*Electronic address: delatorre@mdp.edu.ar

${ }^{\dagger}$ Electronic address: dgoyene@mdp.edu.ar

‡Electronic address: lleitao@mdp.edu.ar
} 
to any of these eigenvalues) but there are strong quantum correlations among them because the observation of one property, say $A_{1}$, forces the appearance of $B_{1}$ although they may be totaly unrelated (like spin and location). In entangled states all sort of astonishing quantum effects appear, like violations of Bell's inequalities, Einstein-Podolsky-Rosen (so called) paradox, Schrödinger cat, nonlocality, contextuality, teleportation, quantum cryptography and computation, etc. The principle of superposition, that generates the entanglement, contains perhaps the central essence of quantum mechanics and almost all pondering concerning the foundations of quantum mechanics involve entangled states.

The opposite to the entangled states are the factorizable states, for instance $\psi_{1}$ or $\psi_{2}$ above, where the properties are objective and the behaviour of the system is closer to classical expectations; for instance, the correlations found are understood as a direct consequence of the preparation of the system. One may erroneously think that there are two classes of states for the quantum system, entangled and factorizable, that correspond to qualitative difference in the behaviour of the system, close to classical in one case and with strong quantum correlations in the other. We will see in this work that this is indeed wrong because factorizable states also exhibit entanglement with respect to other observables. In this sense, all states are entangled; entanglement is not an exceptional feature of some states but is ubiquitous in quantum mechanics.

The fact that factorizability and entanglement are not preserved in a change of the degrees of freedom used to describe the system has been analysed by experts, specially those involved in quantum computation research [1, 2], but this important feature of quantum mechanics is ignored in textbooks, even advanced ones. In this work we present simple calculations that emphasize this remarkable feature and provide thereby a didactic complement for a modern quantum mechanics course. The calculations mentioned would be quite involved without the application of the Quantum Covariance Function that has also received very little consideration in textbooks. In the following sections we will define entanglement and factorizability with rigour and we will prove that every factorizable state becomes entangled in a different factorization of the Hilbert space. For this, we will recall a useful tool provided by the Quantum Covariance Function and we will calculate several explicit examples that may be useful for teachers and students of quantum mechanics at the advanced undergraduate and graduate level.

\section{ENTANGLEMENT IN COMPOUND SYSTEMS}

The state of a compound quantum system, $S=\left(S_{1}, S_{2}\right)$, belongs to a Hilbert space build as the tensor product of spaces corresponding to each subsystem: $\mathcal{H}=\mathcal{H}_{1} \otimes \mathcal{H}_{2}$. This decomposition, denoted as a Tensor Product Structure (TPS), may correspond to two individual physical subsystems like, for instance, one electron and one proton building a hydrogen atom, or to different degrees of freedom or coordinates of one system. The degrees of freedom are expected to be independent in the sense that the assignment of one value to one degree of freedom is compatible with an arbitrary 
assignment of any value for the other one. For quantum mechanics, this means that, in the Hilbert space, the two degrees of freedom $A$ and $B$ will correspond to two observables whose operators act individually in each factor space, that is, they are of the form $A \otimes \mathbb{I}$ and $\mathbb{I} \otimes B$ and therefore they commute. If we define bases in each factor space, $\left\{\varphi_{k}\right\} \in \mathcal{H}_{1}$ and $\left\{\phi_{r}\right\} \in \mathcal{H}_{2}$, the most general state in the Hilbert space is given by an expansion in the basis $\left\{\varphi_{k} \otimes \phi_{r}\right\}$ as

$$
\Psi=\sum_{k, r} C_{k r} \varphi_{k} \otimes \phi_{r}
$$

This state $\Psi$ is factorizable if there exist $\Psi_{1} \in \mathcal{H}_{1}$ and $\Psi_{2} \in \mathcal{H}_{2}$ such that $\Psi=\Psi_{1} \otimes \Psi_{2}$. Otherwise it is entangled. More precisely, we define entanglement by means of Schmidt bi-orthogonal decomposition: for each state $\Psi$ there exist two bases $\left\{\widetilde{\varphi}_{k}\right\} \in \mathcal{H}_{1}$ and $\left\{\widetilde{\phi}_{r}\right\} \in \mathcal{H}_{2}$ such that

$$
\Psi=\sum_{k=1}^{N} \alpha_{k} \widetilde{\varphi}_{k} \otimes \widetilde{\phi}_{k}
$$

where $N \leq \min \left\{D_{1}, D_{2}\right\}$ and where $D_{1}$ and $D_{2}$ are the dimensions of the Hilbert spaces. Notice that in this expansion we do not have a double sum as in the general expansion in Eq.(1). The bases for the bi-orthogonal decomposition are not unique and, of course, depend on the state $\Psi$. If $N=1$ the state is factorizable and if $N \geq 2$ the state is entangled.

Notice that in the determination of whether a state is factorizable or entangled, the factorization of the Hilbert space (that is, the TPS) is crucial and this factorization depends on the choice of the observables corresponding to the degrees of freedom. From the mathematical point of view, every TPS is equivalent but from the physical point of view, the TPS are determined operationally by the measurements and operations that are accessible under given physical circumstances. For instance, if our composite system consists of two particles that are spatially separated, the most natural TPS is given by the tensor product of the single-particle Hilbert spaces associated with the individual particles. However if in this same system, the overall motion is uninteresting and only the relative motion is relevant we may prefer a TPS corresponding, not to the position of the individual particles, but instead, to the center of mass and relative position.

A relevant question is whether some arbitrary state of a system, analysed with different choices of the degrees of freedom, that is, with different TPS, still maintains the property of been factorizable or not. In other words, is factorizability an objective property of the system or is it a feature of our description of the system. In order to approach this question we recall a useful tool provided by the Quantum Covariance Function that relates a state $\Psi$ and two observables $A$ and $B$.

\section{THE QUANTUM COVARIANCE FUNCTION}

Given two arbitrary hermitian operators $A$ and $B$ and a normalized Hilbert space element $\Psi$, we define the Quantum Covariance Function (QCF) by

$$
Q(A, B, \Psi)=\langle\Psi, A B \Psi\rangle-\langle\Psi, A \Psi\rangle\langle\Psi, B \Psi\rangle
$$


This function has been studied in detail[3] and was use to provide an elegant proof of the uncertainty principle in the most general form given by Schrödinger [4]. Another interesting application of this function allows a complete study of manifest and concealed correlations in quantum systems [5]. For our purpose here, we notice that when the observables correspond to two degrees of freedom, that is, they are of the form $A \otimes \mathbb{I}$ and $\mathbb{I} \otimes B$, and the state is factorizable, $\Psi=\Psi_{1} \otimes \Psi_{2}$, then the QCF vanishes. The proof is trivial: $Q\left(A \otimes \mathbb{I}, \mathbb{I} \otimes B, \Psi_{1} \otimes \Psi_{2}\right)=\left\langle\Psi_{1} \otimes \Psi_{2}, A \otimes B \Psi_{1} \otimes\right.$ $\left.\Psi_{2}\right\rangle-\left\langle\Psi_{1}, A \Psi_{1}\right\rangle\left\|\Psi_{2}\right\|^{2}\left\|\Psi_{1}\right\|^{2}\left\langle\Psi_{2}, B \Psi_{2}\right\rangle=0$. Notice that $\Psi$ factorizable implies $Q(A, B, \Psi)=0$ but the inverse is not true: there are cases with vanishing QCF but with entangled states as can be seen in ref. [5]. In any case, if the QCF does not vanish, then we are sure that the state is not factorizable, that is, it is entangled.

\section{TRANSFORMATION ENTANGLEMENT}

Let us consider a quantum system with two subsystems $S=\left(S_{A}, S_{B}\right)$ that may correspond to two degrees of freedom $A$ and $B$. The state of the system belongs then to the Hilbert space $\mathcal{H}=\mathcal{H}_{A} \otimes \mathcal{H}_{B}$ and the two degrees of freedom are represented by operators $A \otimes \mathbb{I}$ and $\mathbb{I} \otimes B$. Let us consider a factorizable, non entangled, state $\Psi=\Psi_{A} \otimes \Psi_{B}$ with $\Psi_{A}$ and $\Psi_{B}$ arbitrary states (not necessarily eigenvectors of $A$ and $B$ ) in the spaces $\mathcal{H}_{A}$ and $\mathcal{H}_{B}$. Then there exists a transformation of the degrees of freedom $F=F(A, B)$ and $G=G(A, B)$ that suggests a different factorization or TPS, $\mathcal{H}=\mathcal{H}_{F} \otimes \mathcal{H}_{G}$, where the state is no longer factorizable: $\Psi \neq \Psi_{F} \otimes \Psi_{G}$ with $\Psi_{F} \in \mathcal{H}_{F}$ and $\Psi_{G} \in \mathcal{H}_{G}$. The state becomes entangled in these new degrees of freedom; the factorizability of states is not invariant under a different factorization of the Hilbert space.

We will next clarify this with two simple examples and we will later give a general proof.

\section{A. System with two coordinates}

Let us consider a very simple system characterized by two space coordinates $X_{1}$ and $X_{2}$. This may correspond to the position of two free particles moving in a line or to one particle moving in a plane (the equivalence of $n$ free particles moving in $\mathbb{R}_{1}$ with one particle moving in $\mathbb{R}_{n}$ is an interesting fact that has been used to relate nonseparability with contextuality [6] ). The state of the system is then an element of $\mathcal{H}_{1} \otimes \mathcal{H}_{2}$ and the two coordinates correspond to the operators $X_{1}=X \otimes \mathbb{I}$ and $X_{2}=\mathbb{I} \otimes X$. If we use the basis $\left\{\varphi_{x}\right\} \in \mathcal{H}_{1}$ and $\left\{\phi_{x}\right\} \in \mathcal{H}_{2}$ corresponding to the eigenvectors of the position operator in both spaces, then the most general factorizable state is given by

$$
\Psi=\left(\int d x_{1} f\left(x_{1}\right) \varphi_{x_{1}}\right) \otimes\left(\int d x_{2} g\left(x_{2}\right) \phi_{x_{2}}\right)=\int d x_{1} \int d x_{2} f\left(x_{1}\right) g\left(x_{2}\right) \varphi_{x_{1}} \otimes \phi_{x_{2}}
$$

where $f(x)$ and $g(x)$ are two properly normalized arbitrary functions. (For more rigour, we should mention that the spaces $\mathcal{H}_{1}$ and $\mathcal{H}_{2}$ are rigged Hilbert spaces that contain not only the normalizable 
square integrable functions but also the improper eigenvectors $\left\{\varphi_{x}\right\}$ and $\left\{\phi_{x}\right\}$. More details on this can be found in advanced quantum mechanics books [7]).

Instead of $X_{1}$ and $X_{2}$ we can now consider another pair of degrees of freedom given by

$$
\begin{aligned}
& A=X_{1}+X_{2}=X \otimes \mathbb{I}+\mathbb{I} \otimes X \\
& B=X_{1}-X_{2}=X \otimes \mathbb{I}-\mathbb{I} \otimes X .
\end{aligned}
$$

Physically, these new coordinates are related to the center of mass and relative distance, in the case of two particles in a line, or to a rotation and reflection of the coordinate axis in the case of one particle moving in a plane. It is a trivial change of variables but, as we will see, with significant consequences for the treatment of the quantum system. Let $\chi_{a, b}$ in $\mathcal{H}_{1} \otimes \mathcal{H}_{2}$ denote the eigenvectors of $A$ and $B$ corresponding to the eigenvalues $a$ and $b$. That is, $A \chi_{a, b}=a \chi_{a, b}$ and $B \chi_{a, b}=b \chi_{a, b}$. One can easily check that $\chi_{a, b}=\varphi_{\frac{1}{2}(a+b)} \otimes \phi_{\frac{1}{2}(a-b)}$. Consider now the degree of freedom $A$ alone, isolated from the other degree of freedom $B$. To this degree of freedom we can associate a Hilbert space $\mathcal{H}_{A}$ spanned by the eigenvectors of $A,\left\{\eta_{a}\right\}$. Similarly, the eigenvectors $\left\{\xi_{b}\right\}$ of $B$, considered independently, generate another Hilbert space $\mathcal{H}_{B}$. The tensor product of these spaces, $\mathcal{H}_{A} \otimes \mathcal{H}_{B}$, provide a different factorization of the Hilbert space of the compound system, spanned by the basis $\left\{\eta_{a} \otimes \xi_{b}\right\}$. The two bases $\left\{\eta_{a} \otimes \xi_{b}\right\}$ and $\left\{\varphi_{x_{1}} \otimes \phi_{x_{2}}\right\}$ are related by

$$
\begin{aligned}
\eta_{a} \otimes \xi_{b} & =\varphi_{\frac{1}{2}(a+b)} \otimes \phi_{\frac{1}{2}(a-b)} \\
\varphi_{x_{1}} \otimes \phi_{x_{2}} & =\eta_{x_{1}+x_{2}} \otimes \xi_{x_{1}-x_{2}} .
\end{aligned}
$$

Notice that this change of basis is trivial, it amounts only to a relabelling or reordering of the basis elements. This is expected because the new and old degrees of freedom commute and therefore they share the same basis. Anyway, if we perform the variable change $x_{1}+x_{2}=a, x_{1}-x_{2}=$ $b, d x_{1} d x_{2}=\frac{1}{2} d a d b$ in the factorizable state in Eq.(44) we get the same state in the new basis given as

$$
\Psi=\frac{1}{2} \iint d a d b f\left(\frac{1}{2}(a+b)\right) g\left(\frac{1}{2}(a-b)\right) \eta_{a} \otimes \xi_{b} .
$$

One can, of course, find many examples of factorizable states that remain factorizable after the change of variables. For instance, the gaussian $f\left(x_{1}\right) g\left(x_{2}\right)=\exp \left[-x_{1}^{2}-x_{2}^{2}\right]$ becomes $\exp \left[\left(-a^{2}-b^{2}\right) / 2\right]$ that is also factorizable, or also a plane wave $\exp \left[i\left(k_{1} x_{1}+k_{2} x_{2}\right]\right.$ remains factorizable. However, not every factorizable state remains so and, in general, $f\left(\frac{1}{2}(a+b)\right) g\left(\frac{1}{2}(a-b)\right) \neq$ $F(a) G(b)$. As an example for nonfactorizability we can take, for instance, a double gaussian for $f\left(x_{1}\right)=\exp \left(-\left(x_{1}-d\right)^{2}\right)+\exp \left(-\left(x_{1}+d\right)^{2}\right)$ and one single gaussian for $g\left(x_{2}\right)=\exp \left(-x_{2}^{2}\right)$.

A more elegant proof that the variable change destroys the factorizability is obtained using the QCF. Clearly, with the factorizable state in Eq.(4), that is with $\Psi=\Psi_{1} \otimes \Psi_{2}$, the QCF vanishes:

$$
\begin{aligned}
Q\left(X_{1}, X_{2}, \Psi\right) & =\langle\Psi, X \otimes X \Psi\rangle-\langle\Psi, X \otimes \mathbb{I} \Psi\rangle\langle\Psi, \mathbb{I} \otimes X \Psi\rangle \\
& =\left\langle\Psi_{1}, X \Psi_{1}\right\rangle\left\langle\Psi_{2}, X \Psi_{2}\right\rangle-\left\langle\Psi_{1}, X \Psi_{1}\right\rangle\left\|\Psi_{2}\right\|^{2}\left\|\Psi_{1}\right\|^{2}\left\langle\Psi_{2}, X \Psi_{2}\right\rangle=0 .
\end{aligned}
$$


However for the same state, a similar calculation gives

$$
\begin{aligned}
Q(A, B, \Psi) & =\left\langle\Psi_{1}, X^{2} \Psi_{1}\right\rangle-\left\langle\Psi_{2}, X^{2} \Psi_{2}\right\rangle-\left\langle\Psi_{1}, X \Psi_{1}\right\rangle^{2}+\left\langle\Psi_{2}, X \Psi_{2}\right\rangle^{2} \\
& =\Delta_{X_{1}}^{2}-\Delta_{X_{2}}^{2} \neq 0
\end{aligned}
$$

because, in general, the "widths" of $\left|f\left(x_{1}\right)\right|^{2}$ and $\left|g\left(x_{2}\right)\right|^{2}$ are different.

Summarizing, a factorizable state in the compound Hilbert space corresponding to the degrees of freedom $X_{1}$ and $X_{2}$ becomes entangled when we consider the degrees of freedom $A=X_{1}+X_{2}$ and $B=X_{1}-X_{2}$ and viceversa. Instead of this simple transformation of the degrees of freedom we can consider any general reversible map between the coordinates $\left(X_{1}, X_{2}\right)$ and $(A, B)$. In this case, the state in Eq.(4) becomes

$$
\Psi=\iint d a d b\left|\frac{\partial\left(x_{1}, x_{2}\right)}{\partial(a, b)}\right| f\left(x_{1}(a, b)\right) g\left(x_{2}(a, b)\right) \eta_{a} \otimes \xi_{b} .
$$

We can prove that, in general, this expression is not factorizable for arbitrary normalized functions $f\left(x_{1}\right)$ and $g\left(x_{2}\right)$. In fact, if there exist functions $F(a)$ and $G(b)$ not vanishing everywhere such that

$$
f\left(x_{1}(a, b)\right) g\left(x_{2}(a, b)\right)=F(a) G(b),
$$

then we reach a contradiction. In order to see this, let us choose, among all possible $f\left(x_{1}\right)$, one that has a zero in $x_{0}$, that is, $f\left(x_{0}\right)=0$. Therefore in the $\left(x_{1}, x_{2}\right)$ plane, the product $f\left(x_{1}\right) g\left(x_{2}\right)$ vanishes along a straight line perpendicular to the $x_{1}$ axis. This line is mapped in the $(a, b)$ plane into a curve given by $x_{1}(a, b)=x_{0}$. We can solve this equation for $a$, that is, $a=a_{0}(b)$ and replace it in the right hand side of Eq.(13) obtaining $F\left(a_{0}(b)\right) G(b)=0 \forall b$. Now, since $\forall b, G(b) \neq 0$ by definition, we must have $F\left(a_{0}(b)\right)=0 \forall b$, in contradiction with the assumption that $F$ does not vanishes everywhere.

From the example seen, it becomes clear that every change to new degrees of freedom that mixes the old ones, destroys the factorization of the state. The only possibility to preserve the factorization is when the degrees of freedom are not mixed, that is, the transformations are of the type $A=A\left(X_{1}\right)$ and $B=B\left(X_{2}\right)$. Therefore, the unitary transformations in the Hilbert space $\mathcal{H}_{1} \otimes \mathcal{H}_{2}$ that leave the TPS invariant are of the type $U_{A} \otimes U_{B}$, for instance, the time evolution of internal and external degrees of freedom[2]. A generalization of this to TPS involving any number of factors is evident.

\section{B. System with two spins}

In this example, let us consider a system of two particles with spin $1 / 2$. As degrees of freedom to characterize the system we can take, as is usually done, the $z$ component of both spins $S_{1 z}$ and $S_{2 z}$. Their corresponding two dimensional Hilbert spaces $\mathcal{H}_{1}$ and $\mathcal{H}_{2}$ are spanned by the two basis 
$\left\{\varphi_{ \pm}\right\}$and $\left\{\phi_{ \pm}\right\}$. The four dimensional Hilbert space $\mathcal{H}=\mathcal{H}_{1} \otimes \mathcal{H}_{2}$ for the system has a basis $\left\{\psi_{k, r}=\varphi_{k} \otimes \phi_{r}, k, r=+,-\right\}$ and the most general factorizable state is given by

$$
\Psi=\Psi_{1} \otimes \Psi_{2}=\left(\sum_{k} \alpha_{k} \varphi_{k}\right) \otimes\left(\sum_{r} \beta_{r} \phi_{r}\right)=\sum_{k, r} \alpha_{k} \beta_{r} \psi_{k, r} .
$$

We will see that when we factorize the Hilbert space corresponding to other degrees of freedom this state becomes entangled.

As different degrees of freedom we can take, for instance, the square of two orthogonal components of total spin.

$$
\begin{aligned}
& S_{z}^{2}=\left(S_{z} \otimes \mathbb{I}+\mathbb{I} \otimes S_{z}\right)^{2}=\frac{\hbar^{2}}{2} \mathbb{I} \otimes \mathbb{I}+2 S_{z} \otimes S_{z} \\
& S_{x}^{2}=\left(S_{x} \otimes \mathbb{I}+\mathbb{I} \otimes S_{x}\right)^{2}=\frac{\hbar^{2}}{2} \mathbb{I} \otimes \mathbb{I}+2 S_{x} \otimes S_{x} .
\end{aligned}
$$

These two operators commute $\left[S_{z}^{2}, S_{x}^{2}\right]=0$ and their eigenvalues are 0 and $\hbar^{2}$ corresponding to the degeneracy eigenvectors $\left\{\chi_{s, t}, s, t=0,1\right\}$. That is,

$$
\begin{aligned}
S_{z}^{2} \chi_{s, t} & =s \hbar^{2} \chi_{s, t} \\
S_{x}^{2} \chi_{s, t} & =t \hbar^{2} \chi_{s, t}, s, t=0,1 .
\end{aligned}
$$

We have then two different bases $\left\{\psi_{k, r}, k, r=+,-\right\}$ and $\left\{\chi_{s, t}, s, t=0,1\right\}$. In this example, the new and old degrees of freedom do not commute and they do not share the basis as was the case in the previous example. The unitary transformation relating both bases is

$$
\left(\begin{array}{l}
\chi_{1,1} \\
\chi_{1,0} \\
\chi_{0,1} \\
\chi_{0,0}
\end{array}\right)=\frac{1}{\sqrt{2}}\left(\begin{array}{cccc}
1 & 1 & 0 & 0 \\
1 & -1 & 0 & 0 \\
0 & 0 & 1 & 1 \\
0 & 0 & 1 & -1
\end{array}\right)\left(\begin{array}{c}
\psi_{+,+} \\
\psi_{-,-} \\
\psi_{+,-} \\
\psi_{-,+}
\end{array}\right) .
$$

Now, if we take the most general factorizable state in the TPS related with the basis $\left\{\psi_{k, r}\right\}$, given in Eq.(14), and we make the change of basis with the unitary transformation, then one can prove that the resulting expression is, in general, not factorizable in the TPS related to $\left\{\chi_{s, t}\right\}$, that is, it can not always be written in the form

$$
\Psi=\sum_{s, t} \delta_{s} \eta_{t} \chi_{s, t}
$$

The change of basis destroys the factorizability. Instead of this explicit long calculation it is simpler to use the QCF to show that the state is entangled in the Hilbert space factorization corresponding to the new variables. Doing this we obtain

$$
Q\left(S_{z}^{2}, S_{x}^{2}, \Psi\right)=-\hbar^{2}\left\langle\Psi_{1}, S_{y} \Psi_{1}\right\rangle\left\langle\Psi_{2}, S_{y} \Psi_{2}\right\rangle-4\left\langle\Psi_{1}, S_{x} \Psi_{1}\right\rangle\left\langle\Psi_{2}, S_{x} \Psi_{2}\right\rangle\left\langle\Psi_{1}, S_{z} \Psi_{1}\right\rangle\left\langle\Psi_{2}, S_{z} \Psi_{2}\right\rangle \neq 0
$$

for arbitrary $\Psi_{1}$ and $\Psi_{2}$. 


\section{General proof}

The simple example concerning two spatial coordinates can be generalized to provide a general proof. Let us consider a quantum system with two subsystems $S=\left(S_{A}, S_{B}\right)$ described in the Hilbert space $\mathcal{H}=\mathcal{H}_{A} \otimes \mathcal{H}_{B}$ corresponding to the TPS associated with the two degrees of freedom $A \otimes \mathbb{I}$ and $\mathbb{I} \otimes B$. Let us assume a factorizable, non entangled, normalized state $\Psi=\Psi_{A} \otimes \Psi_{B}$ where $\Psi_{A}$ and $\Psi_{B}$ are two arbitrary normalized states in the factor spaces $\mathcal{H}_{A}$ and $\mathcal{H}_{B}$. Then there exists a transformation of the degrees of freedom, $F=A \otimes \mathbb{I}+\mathbb{I} \otimes B$ and $G=A \otimes \mathbb{I}-\mathbb{I} \otimes B$, whose TPS has a different factorization, $\mathcal{H}=\mathcal{H}_{F} \otimes \mathcal{H}_{G}$, where the state is no longer factorizable. In order to prove this we show that the $\mathrm{QCF} Q(F, G, \Psi) \neq 0$ and therefore the state $\Psi$ is entangled in the Hilbert space factorization $\mathcal{H}=\mathcal{H}_{F} \otimes \mathcal{H}_{G}$ corresponding to the degrees of freedom $F$ and $G$. Notice that $F G=(A \otimes \mathbb{I}+\mathbb{I} \otimes B)(A \otimes \mathbb{I}-\mathbb{I} \otimes B)=A^{2} \otimes \mathbb{I}-\mathbb{I} \otimes B^{2}$ and then we have

$$
\begin{aligned}
Q(F, G, \Psi)= & \left\langle\Psi_{A} \otimes \Psi_{B},\left(A^{2} \otimes \mathbb{I}-\mathbb{I} \otimes B^{2}\right) \Psi_{A} \otimes \Psi_{B}\right\rangle- \\
& \left\langle\Psi_{A} \otimes \Psi_{B},(A \otimes \mathbb{I}+\mathbb{I} \otimes B) \Psi_{A} \otimes \Psi_{B}\right\rangle\left\langle\Psi_{A} \otimes \Psi_{B},(A \otimes \mathbb{I}-\mathbb{I} \otimes B) \Psi_{A} \otimes \Psi_{B}\right\rangle \\
= & \left\langle\Psi_{A}, A^{2} \Psi_{A}\right\rangle-\left\langle\Psi_{B}, B^{2} \Psi_{B}\right\rangle-\left\langle\Psi_{A}, A \Psi_{A}\right\rangle^{2}+\left\langle\Psi_{B}, B \Psi_{B}\right\rangle^{2} \\
= & \Delta_{A}^{2}-\Delta_{B}^{2} \neq 0
\end{aligned}
$$

because the indeterminacies of $A$ and $B$ in the arbitrary states $\Psi_{A}$ and $\Psi_{B}$ are, in general, different.

\section{CONCLUSIONS}

We have seen that the factorizability of a state is a property that is not invariant under a change of the degrees of freedom that we use in order to describe the system. This proof is made simple by the use of the QCF whose non-vanishing is a criterium for entanglement.

The fact that the appearance of entanglement depends on the choice of degrees of freedom can find an interesting application in the "disentanglement" of a state. One can, sometimes, transform an entangled state into a factorizable one by a judicious choice of the degrees of freedom. In some sense this is the inverse problem to the one presented in section IV. One example of this is provided in Ref. [8] where the entangled state of the compound system of one proton and one electron with Coulomb interaction becomes factorizable when we use center of mass and relative position coordinates instead of the individual spacial coordinates of the proton and electron. In this case, the two particle system consisting in one proton and one electron in an entangled state is described in a simpler, factorizable, state of two fictitious noninteracting particles: one with the total mass of the hydrogen atom, moving freely in space, and another particle with the effective mass moving in a fixed Coulomb potential.

Perhaps the most important manifestation of quantum correlations, that is, those that can not be explained in terms of some classical interaction, involves the violations of Bell's inequalities. Furthermore, it has been shown[9] that in every nonfactorizable or entangled state there are ob- 
servables that violate Bell's inequalities. In this work, we have seen that for any system in a factorizable state, we can find different degrees of freedom that suggest a different factorization of the Hilbert space where the same state becomes entangled. As a consequence of this we can conclude that in every state, even for those factorizable, we can find pairs of observables that will violate Bell's inequalities. This violation of the classical behaviour is then not exceptional but is ubiquitous in quantum systems.

[1] P. Zanardi, D. A. Lidar and S. Lloyd, "Quantum Tensor Product Structures are Observable Induced" Phys, Rev. Lett. 92 060402, (2004).

[2] N. L. Harshman, S. Wickramasekara, "Tensor Product Structures, Entanglement, and Particle Scattering" Open Sys. and Inf. Dyn. 14,341-351, (2007). N. L. Harshman, S. Wickramasekara, "Galilean and Dynamical Invariance of Entanglement in Particle Scattering" Phys, Rev. Lett. 92 060402, (2004).

[3] A. C. de la Torre, P. Catuogno, S. Ferrando, "Uncertainty and nonseparability", Found. of Phys. Lett. 2, 235-244, (1991).

[4] E. Schrödinger, "Zum Heisenbergschen Unschärfeprinzip" Berliner Berichte 296303 (1930).

[5] A. C. de la Torre, J. L. Iguain, "Manifest and concealed correlations in quantum mechanics" Eur. J. Phys. 19, 563568, (1998).

[6] A. C. de la Torre, "Contextuality in quantum systems" Am. J. Phys. 62, 808-812, (1994).

[7] A simple presentation of this mathematics can be found in Sec. 1.4 of L. E. Ballentine, "Quantum Mechanics. A Modern Development", World Scientifc, Singapore, (1998).

[8] P. Tommasini, E. Timmermans and A. F. R. de Toledo Piza "The hydrogen atom as an entangled electron-proton system" Am. J. Phys. 66, 881-886 (1998).

[9] N. Gisin, "Bell's inequality holds for all non-product states" Phys. Lett. A 154, 201202, (1991). 